\title{
Oscillation Criteria for Linear Neutral Delay Differential Equations of First Order
}

\author{
Fatima N. Ahmed, Rokiah Rozita Ahmad, \\ Ummul Khair Salma Din, and Mohd Salmi Md Noorani
}

School of Mathematical Sciences, Faculty of Science and Technology, Universiti Kebangsaan Malaysia, 43600 Bangi, Selangor, Malaysia

Correspondence should be addressed to Fatima N. Ahmed; zahra80zahra@yahoo.com

Received 10 May 2013; Revised 11 August 2013; Accepted 12 August 2013

Academic Editor: Aref Jeribi

Copyright (C) 2013 Fatima N. Ahmed et al. This is an open access article distributed under the Creative Commons Attribution License, which permits unrestricted use, distribution, and reproduction in any medium, provided the original work is properly cited.

Some new sufficient conditions for oscillation of all solutions of the first-order linear neutral delay differential equations are obtained. Our new results improve many well-known results in the literature. Some examples are inserted to illustrate our results.

\section{Introduction}

A neutral delay differential equation (NDDE) is a differential equation in which the highest-order derivative of the unknown function is evaluated both at the present state at time $t$ and at the past state at time $t-k$ for some positive constant $k$.

In the last two decades, there has been an increasing interest in obtaining sufficient conditions for the oscillation and/or nonoscillation of solutions of neutral delay differential equations. Particularly, we mention the papers by Ladas and Sficas [1], Chuanxi and Ladas [2], Ruan [3], Elabbasy and Saker [4], Kulenović et al. [5], and Karpuz and Öcalan [6] who investigated NDDEs with variable coefficients. To a large extent, this is due to its theoretical interest as well as to its importance in applications. It suffices to note that NDDEs appear in the study of networks containing lossless transmission lines (as in high-speed computers where the lossless transmission lines are used to interconnect switching circuits) in population dynamics and also in many applications in epidemics and infection diseases. We refer reader to [1-18] for relevant studies on this subject.

In this paper, we consider the linear first-order NDDE of the type

$$
\begin{aligned}
(x(t) & -p x(t-\tau))^{\prime}+q_{1} x(t) \\
& +q_{2}(t) x(t-\sigma)=0 ; \quad t \geq t_{0},
\end{aligned}
$$

where $p, q_{1}, \tau, \sigma \in(0, \infty)$ and $q_{2}(t) \in C\left[\left[t_{0}, \infty\right), \mathbb{R}\right]$. When $q_{1} \equiv 0$ and $q_{2}(t)=q, q$ is a constant, Jaroš [9] established some new oscillation conditions for all solutions of (1), and his technique was based on the study of the characteristic equation

$$
\lambda-\lambda p e^{-\lambda \tau}+q e^{-\lambda \sigma}=0 .
$$

Zhang [19], Ladas and Sficas [1], Grammatikopoulos et al. [10], and Yu et al. [8] considered (1) when $q_{1} \equiv 0$, and they obtained some sufficient conditions for oscillation of (1). The purpose of this work is to present some new sufficient conditions under which all solutions of (1) are oscillatory. In order to achieve this object, we are first concerned with $\operatorname{NDDE}$ (1) with constant coefficients (when $q_{2}(t) \equiv q_{2}, q_{2}$ is a constant). That is,

$$
\begin{aligned}
& (x(t)-p x(t-\tau))^{\prime}+q_{1} x(t) \\
& \quad+q_{2} x(t-\sigma)=0, \quad t \geq 0 .
\end{aligned}
$$

Some illustrating examples are given. In some sense, the established results extend and improve some previous investigations such as $[1,8-10,19]$.

As usual, a solution of (1) is said to be oscillatory if it has arbitrarily large zeros and nonoscillatory if it is eventually positive or eventually negative. A function $x(t)$ is called eventually positive (or negative) if there exists $t_{0}$ such 
that $x(t)>0 \quad($ or $x(t)<0)$ for all $t \geq t_{0}$. Equation (1) is called oscillatory if all its solutions are oscillatory; otherwise, it is called nonoscillatory.

\section{Main Results}

In this section, we give some new sufficient conditions for the oscillation of all solutions of (1) and (3). This is done by using the following well-known lemmas which are from $[11,12]$.

Lemma 1. Consider the NDDE

$$
(x(t)+p x(t-\tau))^{\prime}+\sum_{i=1}^{n} q_{i} x\left(t-\sigma_{i}\right)=0, \quad t \geq t_{0},
$$

where $\tau \geq 0, q_{i}>0$, and $\sigma_{i} \geq 0$ for all $i=1,2, \ldots, n$.

Let $x(t)$ be a positive solution of (4). Set

$$
z(t)=x(t)-p x(t-\tau) .
$$

If $p \geq-1$, then $z(t)$ is a positive and decreasing solution of (4); that is,

$$
z^{\prime}(t)+p z^{\prime}(t-\tau)+\sum_{i=1}^{n} q_{i} z\left(t-\sigma_{i}\right)=0, \quad t \geq t_{0} .
$$

Lemma 2. Let $p$ and $\tau$ be positive constants. Let $x(t)$ be an eventually positive solution of the delay differential inequality

$$
x^{\prime}(t)+p x(t-\tau) \leq 0
$$

Then for t sufficiently large,

$$
x(t-\tau) \leq B x(t),
$$

where

$$
B=\frac{4}{(p \tau)^{2}}
$$

Our main results can now be given as follows.

Theorem 3. Consider NDDE (3). Assume that

(i) $q_{2} \in[0, \infty), \sigma \geq \tau, 0<p e^{q_{1} \tau}<1$ and

(ii) $\tau\left(q_{1} p e^{q_{1} \tau}+q_{2} e^{q_{1} \sigma}\right)>\left(1-p e^{q_{1} \tau} \bar{m}\right)^{2} / \bar{m}$,

where $\bar{m}$ is the unique real root of the equation

$$
1-p e^{q_{1} \tau} m=\ln m, \quad 1 \leq m \leq \frac{1}{p^{e^{-q_{1} \tau}}} .
$$

Then all solutions of (3) are oscillatory.

Proof. Assume, for the sake of a contradiction, that (3) has a nonoscillatory solution $x(t)$. Without loss of generality, assume that $x(t)>0 \quad \forall t \geq t_{0}>0$. Let

$$
x(t)=e^{-q_{1} t} y(t) .
$$

So that $y(t)$ is also a positive solution of (3).
That is,

$$
\left(y(t)-p_{1} y(t-\tau)\right)^{\prime}+\sum_{i=1}^{2} a_{i} y\left(t-\tau_{i}\right)=0,
$$

where

$$
\begin{gathered}
p_{1}=p e^{q_{1} \tau}, \quad a_{1}=q_{1} p e^{q_{1} \tau}, \quad a_{2}=q_{2} e^{q_{1} \sigma}, \\
\tau_{1}=\tau, \quad \tau_{2}=\sigma .
\end{gathered}
$$

Set for $t \geq t_{0}+2 \tau$

$$
\begin{gathered}
z(t)=y(t)-p_{1} y(t-\tau), \\
w(t)=\frac{z(t-\tau)}{z(t)} .
\end{gathered}
$$

Thus it follows from Lemma 1 that $z(t)$ is a positive and decreasing solution of

$$
z^{\prime}(t)-p_{1} z^{\prime}(t-\tau)+\sum_{i=1}^{2} a_{i} z\left(t-\tau_{i}\right)=0,
$$

and in particular (as $\sigma>\tau$ implies that $t-\tau_{i} \leq t-\tau, i=1,2$ ), it follows that

$$
z^{\prime}(t)-p_{1} z^{\prime}(t-\tau)+\left(a_{1}+a_{2}\right) z(t-\tau) \leq 0 .
$$

But we have

$$
z^{\prime}(t-\tau)<0
$$

This implies that

$$
z^{\prime}(t)+\left(a_{1}+a_{2}\right) z(t-\tau) \leq 0 .
$$

Applying Lemma 2 with (18) we get

$$
z(t-\tau) \leq B z(t) ; \quad B=\frac{4}{\tau^{2}\left(a_{1}+a_{2}\right)^{2}} .
$$

Then $w(t)$ is bounded.

Dividing (16) by $z(t)>0$ and integrating from $t-\tau$ to $t$, we get

$$
\begin{aligned}
\ln w(t) & \geq\left(a_{1}+a_{2}\right) \int_{t-\tau}^{t} w(s) d s \\
& -p_{1} \int_{t-\tau}^{t} w(s) \frac{d}{d s}(\ln z(s-\tau)) d s .
\end{aligned}
$$

Let $m=\lim _{t \rightarrow \infty}$ inf $w(t)$.

Then, it follows from (20) that for $\varepsilon>0$ and sufficiently small,

$$
\ln (m+\varepsilon) \geq\left(a_{1}+a_{2}\right)(m-\varepsilon) \tau+p_{1}(m-\varepsilon) \ln (m-\varepsilon) .
$$

As $\varepsilon$ is arbitrary, so we have

$$
\left(a_{1}+a_{2}\right) \tau \leq \frac{\left(1-p_{1} m\right) \ln m}{m} .
$$


Let

$$
F(m)=\frac{\left(1-p_{1} m\right) \ln m}{m}, \quad 1 \leq m \leq B .
$$

Then

$$
F^{\prime}(m)=\frac{1-p_{1} m-\ln m}{m^{2}}, \quad 1 \leq m \leq B .
$$

Let $\bar{m}$ be the unique real root of the equation

$$
1-p_{1} m=\ln m, \quad m \in\left[1, \frac{1}{p_{1}}\right] .
$$

Then

$$
\max _{m \geq 1} F(m)=F(\bar{m})=\frac{\left(1-p_{1} \bar{m}\right)^{2}}{\bar{m}} .
$$

Hence

$$
\left(q_{1} p e^{q_{1} \tau}+q_{2} e^{q_{1} \sigma}\right) \tau \leq \frac{\left(1-p e^{q_{1} \tau} \bar{m}\right)^{2}}{\bar{m}} .
$$

This contradicts condition (ii) and then completes the proof.

Example 4. Consider the NDDE

$$
\begin{aligned}
(x(t) & \left.-\frac{1}{9} x\left(t-\frac{\pi}{2}\right)\right)^{\prime}+\frac{1}{9} x(t) \\
+x\left(t-\frac{5 \pi}{2}\right) & =0, \quad t \geq 0 .
\end{aligned}
$$

We note that

$$
p=\frac{1}{9}, \quad q_{1}=\frac{1}{9}, \quad q_{2}=1, \quad \tau=\frac{\pi}{2}, \quad \sigma=\frac{5 \pi}{2} .
$$

Then we have

(i) $0<p e^{q_{1} \tau}=(1 / 9) e^{\pi / 18}<1$ and $\sigma \geq \tau$,

(ii)

$$
\begin{aligned}
\tau\left(q_{1} p e^{q_{1} \tau}+q_{2} e^{q_{1} \sigma}\right) & =\frac{\pi}{2}\left(\frac{1}{81} e^{\pi / 18}+\frac{1}{9} e^{5 \pi / 18}\right) \\
& >\frac{\left(1-p e^{q_{1} \tau} \bar{m}\right)^{2}}{\bar{m}}=\frac{\left(1-(1 / 9) e^{\pi / 18}\right)^{2}}{2},
\end{aligned}
$$

where $\bar{m}=2$ is the unique real root of the equation

$$
1-\frac{1}{9} e^{\pi / 18} m=\ln m, \quad m \in\left[1,9 e^{-\pi / 18}\right] .
$$

Then all the hypotheses of Theorem 3 are satisfied, and therefore every solution of (28) oscillates. (Indeed $x(t)=$ $\sin t$ is such a solution.)
Theorem 5. Consider the NDDE (1). Assume that

(iii) $0<p e^{q_{1} \tau}<1, \sigma=\tau$, and $q_{2}(t) \in C\left[\left[t_{0}, \infty\right)\right.$, $(0, \infty)$ ] is periodic with period $\tau$,

(iv) $\lim _{t \rightarrow \infty} \inf \int_{t-\tau}^{t} e^{q_{1} \tau}\left(q_{2}(s)+q_{1} p\right) d s>\left(1-p e^{q_{1} \tau} \bar{m}\right)^{2}$ $/ \bar{m}$

where $\bar{m}$ is defined as in Theorem 3. Then all solutions of (1) are oscillatory.

Proof. Assume, for the sake of contradiction, that (1) has a nonoscillatory solution $x(t)$. Without loss of generality, assume that $x(t)>0 \quad \forall t \geq t_{0}>0$. Let

$$
x(t)=e^{-q_{1} t} y(t),
$$

which is oscillation invariant transformation. Then $y(t)$ is a positive solution of the equation

$$
\left(y(t)-p_{1} y(t-\tau)\right)^{\prime}+q(t) y(t-\tau)=0,
$$

where $p_{1}=p e^{q_{1} \tau}$ and $q(t)=e^{q_{1} \tau}\left(q_{2}(t)+q_{1} p\right)$ is periodic with period $\tau$.

Let

$$
z(t)=y(t)-p_{1} y(t-\tau)
$$

Then $z(t)$ is decreasing positive solution of the equation

$$
\left(z(t)-p_{1} z(t-\tau)\right)^{\prime}+q(t) z(t-\tau)=0 .
$$

Set

$$
w(t)=\frac{z(t-\tau)}{z(t)} .
$$

This implies that $w(t) \geq 1$, since $z(t-\tau) \geq z(t)$.

Dividing both sides of (33) by $z(t)$ and then integrating from $t-\tau$ to $t$, we obtain that

$\ln w(t)$

$$
=\int_{t-\tau}^{t} \frac{q(s)\left(y(s-\tau)-p_{1} y(s-2 \tau)+p_{1} y(s-2 \tau)\right)}{y(s)-p_{1} y(s-\tau)} d s .
$$

Hence

$\ln w(t)$

$$
=\int_{t-\tau}^{t} q(s) w(s) d s+p_{1} \int_{t-\tau}^{t}\left(\frac{q(s) y(s-2 \tau)}{y(s)-p_{1} y(s-\tau)}\right) d s .
$$

Since $q(t)$ is periodic with period $\tau$, then we obtain

$$
q(t)=q(t-\tau)=-\frac{\left(y(t-\tau)-p_{1} y(t-2 \tau)\right)^{\prime}}{y(t-2 \tau)} .
$$


Substituting in (38) we find, for all $t \geq t_{0}$,

$$
\begin{aligned}
\ln w(t)= & \int_{t-\tau}^{t} q(s) w(s) d s \\
& -p_{1} \int_{t-\tau}^{t} w(s) d \ln \left(y(s-\tau)-p_{1} y(s-2 \tau)\right) d s .
\end{aligned}
$$

Now, we want to prove that $w(t)$ is bounded.

Applying the assumption (iv), we can find $t^{*} \in(t-\tau, t)$ such that

$$
\int_{t-\tau}^{t^{*}} q(s) d s>\frac{F(\bar{m})}{2}, \quad \int_{t^{*}}^{t} q(s) d s>\frac{F(\bar{m})}{2},
$$

where $F(\bar{m})$ is similar as in the proof of Theorem 3 .

Integrating (33) from $t^{*}$ to $t$ we obtain

$$
\begin{aligned}
y\left(t^{*}\right)-p_{1} y\left(t^{*}-\tau\right) & \geq \int_{t^{*}}^{t} q(s) y(s-\tau) d s \\
& \geq \int_{t^{*}}^{t} q(s)\left(y(s-\tau)-p_{1} y(s-2 \tau)\right) d s,
\end{aligned}
$$

Using Bonnet's Theorem and in particular (as $z^{\prime}(t-\tau)<o$ ), we get

$$
\begin{aligned}
y\left(t^{*}\right) & -p_{1} y\left(t^{*}-\tau\right) \\
& \geq\left[y(t-\tau)-p_{1} y(t-2 \tau)\right] \cdot \int_{t^{*}}^{t} q(s) d s .
\end{aligned}
$$

Integrating (33) from $t-\tau$ to $t^{*}$, we get

$$
\begin{aligned}
y(t-\tau)-p_{1} y(t-2 \tau) \\
\quad \geq \int_{t-\tau}^{t^{*}} q(s) y(s-\tau) d s \\
\quad \geq \int_{t-\tau}^{t^{*}} q(s)\left(y(s-\tau)-p_{1} y(s-2 \tau)\right) d s .
\end{aligned}
$$

Using Bonnet's Theorem and in particular (as $z^{\prime}(t-\tau)<o$ ), we get

$$
\begin{aligned}
& y(t-\tau)-p_{1} y(t-2 \tau) \\
& \quad \geq\left[y\left(t^{*}-\tau\right)-p_{1} y\left(t^{*}-2 \tau\right)\right] \cdot \int_{t-\tau}^{t^{*}} q(s) d s .
\end{aligned}
$$

Combining (43) and (45), we conclude

$$
\begin{aligned}
y\left(t^{*}\right) & -p_{1} y\left(t^{*}-\tau\right) \\
& \geq\left(y\left(t^{*}-\tau\right)-p_{1} y\left(t^{*}-2 \tau\right)\right)\left(\frac{F(\bar{m})}{2}\right)^{2},
\end{aligned}
$$

or

$$
w\left(t^{*}\right)=\frac{y\left(t^{*}-\tau\right)-p_{1} y\left(t^{*}-2 \tau\right)}{y\left(t^{*}\right)-p_{1} y\left(t^{*}-\tau\right)} \leq \frac{4}{(F(\bar{m}))^{2}} .
$$

Then $w(t)$ is bounded.
Now, let

$$
m=\lim _{t \rightarrow \infty} \inf w(t) .
$$

But we have proved that $w(t)$ is bounded; that is, $m$ is finite. From (40), we obtain

$$
\ln m \geq p_{1} m \ln m+\lim _{t \rightarrow \infty} \inf m \int_{t-\tau}^{t} q(s) d s .
$$

Therefore, we get

$$
\lim _{t \rightarrow \infty} \inf m \int_{t-\tau}^{t} q(s) d s \leq \frac{1-p_{1} m}{m} \ln m .
$$

Hence

$$
\lim _{t \rightarrow \infty} \inf \int_{t-\tau}^{t} q(s) d s \leq \frac{\left(1-p_{1} \bar{m}\right)^{2}}{\bar{m}} .
$$

This contradicts our assumption (iv) and then completes the proof.

Example 6. Consider the NDDE

$$
\begin{aligned}
(x(t) & \left.-\frac{1}{9} x(t-\pi)\right)^{\prime}+\frac{1}{18} x(t) \\
& +(1+\cos 2 t) x(t-\pi)=0, \quad t \geq 0,
\end{aligned}
$$

where

$$
\begin{gathered}
p=\frac{1}{9}, \quad q_{1}=\frac{1}{18}, \quad \tau=\sigma=\pi, \\
q_{2}(t)=1+\cos 2 t .
\end{gathered}
$$

Then we have

(1) $0<p e^{q_{1} \tau}=e^{\pi / 18} / 9<1$;

(2) $q_{2}(t)=1+\cos 2 t \in C[[0, \infty),(0, \infty)]$ is periodic with period $\pi$ and satisfies

$$
\begin{aligned}
\lim _{t \rightarrow \infty} \inf & \int_{t-\tau}^{t} e^{q_{1} \tau}\left(q_{2}(s)+q_{1} p\right) d s \\
& =\lim _{t \rightarrow \infty} \inf \int_{t-\pi}^{t} e^{\pi / 18}\left((1+\cos 2 s)+\frac{1}{162}\right) d s \\
& =e^{\pi / 18} \lim _{t \rightarrow \infty} \inf \left(s+\frac{s}{162}+\left.\frac{1}{2} \sin 2 s\right|_{t-\pi} ^{t}\right) \\
& =\infty>\frac{\left(1-p e^{q_{1} \tau} \bar{m}\right)^{2}}{\bar{m}}=\frac{\left(1-(2 / 9) e^{\pi / 18}\right)^{2}}{2},
\end{aligned}
$$

where $\bar{m}=2$ is the unique real root of the equation

$$
1-\frac{1}{9} e^{\pi / 18} m=\ln m ; \quad 1 \leq m \leq 9 e^{-\pi / 18} .
$$

Therefore (52) satisfies all the hypotheses of Theorem 5. Hence every solution of this equation is oscillatory. 
Theorem 7. Suppose that condition (iii) holds. If

(v) $\lim _{t \rightarrow \infty} \inf \int_{t-\tau}^{t} e^{q_{1} \tau}\left[q_{2}(s)+q_{1} p\right] d s>\left(1-p e^{q_{1} \tau}\right) / e$, then every solution of (1) is oscillatory.

Proof. Proceeding as in the proof of Theorem 5, we get (49) which implies that

$$
\ln m \geq p_{1} \ln m+m \lim _{t \rightarrow \infty} \inf \int_{t-\tau}^{t} q(s) d s .
$$

Hence

$$
\lim _{t \rightarrow \infty} \inf \int_{t-\tau}^{t} q(s) d s \leq \frac{1-p_{1}}{m} \ln m \leq \frac{1-p_{1}}{e} .
$$

But this is a contradiction of assumption (v), and then the proof is complete.

Example 8. Consider the NDDE

$$
\begin{aligned}
(x(t) & \left.-\frac{1}{5 e} x\left(t-\frac{\pi}{2}\right)\right)^{\prime}+\frac{2}{\pi} x(t) \\
& +(e+\sin 4 t) x\left(t-\frac{\pi}{2}\right)=0, \quad t \geq 0 .
\end{aligned}
$$

Here we have

$$
\begin{aligned}
p=\frac{1}{5 e}, \quad q_{1} & =\frac{2}{\pi}, \quad \tau=\sigma=\frac{\pi}{2}, \\
q_{2}(t) & =e+\sin 4 t .
\end{aligned}
$$

Note that $q_{2}(t)=e+\sin 4 t$ is positive and periodic with period $\pi / 2$, and also

(1) $0<p e^{q_{1} \tau}=1 / 5<1$,

(2)

$$
\begin{aligned}
& \lim _{t \rightarrow \infty} \inf \int_{t-\tau}^{t} e^{q_{1} \tau}\left[q_{2}(s)+q_{1} p\right] d s \\
& \quad=\lim _{t \rightarrow \infty} \inf \int_{t-\tau}^{t} e\left[(e+\sin 4 t)+\frac{2}{5 e \pi}\right] d s=\infty \\
& >\frac{\left(1-p e^{q_{1} \tau}\right)}{e}=\frac{4}{5 e} .
\end{aligned}
$$

Then (58) satisfies hypotheses of Theorem 7, and so all its solutions are oscillatory.

\section{Funding}

This research has been completed with the support of these Grants: ukm-DLP-2011-049, DIP-2012-31, and FRGS/1/ 2012/SG04/ukm/01/1.

\section{References}

[1] G. Ladas and Y. G. Sficas, "Oscillations of neutral delay differential equations," Canadian Mathematical Bulletin, vol. 29, no. 4, pp. 438-445, 1986.
[2] Q. Chuanxi and G. Ladas, "Oscillations of first-order neutral equations with variable coefficients," Monatshefte für Mathematik, vol. 109, no. 2, pp. 103-111, 1990.

[3] S. G. Ruan, "Oscillations for first order neutral differential equations with variable coefficients," Bulletin of the Australian Mathematical Society, vol. 43, no. 1, pp. 147-152, 1991.

[4] E. M. Elabbasy and S. H. Saker, "Oscillation of delay differential equation with several positive and negative coefficients," Discussiones Mathematicae, vol. 23, pp. 39-52, 2003.

[5] M. R. S. Kulenović, G. Ladas, and A. Meimaridou, "Necessary and sufficient condition for oscillations of neutral differential equations," Australian Mathematical Society Journal B, vol. 28, no. 3, pp. 362-375, 1987.

[6] B. Karpuz and O. Öcalan, "Oscillation criteria for some classes of linear delay differential equations of first-order," Bulletin of the Institute of Mathematics. Academia Sinica, vol. 3, no. 2, pp. 293-314, 2008.

[7] I. R. Al-Amri, "On the oscillation of first-order neutral delay differential equations with real coefficients," International Journal of Mathematics and Mathematical Sciences, vol. 29, no. 4, pp. 245-249, 2002.

[8] J. S. Yu, Z. Wang, and C. X. Qian, "Oscillation of neutral delay differential equations," Bulletin of the Australian Mathematical Society, vol. 45, no. 2, pp. 195-200, 1992.

[9] J. Jaroš, "On characterization of oscillations in first-order linear neutral differential equations," Funkcialaj Ekvacioj, vol. 34, no. 2, pp. 331-342, 1991.

[10] M. K. Grammatikopoulos, E. A. Grove, and G. Ladas, "Oscillations of first-order neutral delay differential equations," Journal of Mathematical Analysis and Applications, vol. 120, no. 2, pp. 510-520, 1986.

[11] I. Győri and G. Ladas, Oscillation Theory of Delay Differential Equations, Oxford Mathematical Monographs, The Clarendon Press, New York, NY, USA, 1991.

[12] I. Kubiaczyk and S. H. Saker, "Oscillation of solutions to neutral delay differential equations," Mathematica Slovaca, vol. 52, no. 3, pp. 343-359, 2002.

[13] R. D. Driver, "A mixed neutral system," Nonlinear Analysis. Theory, Methods \& Applications, vol. 8, no. 2, pp. 155-158, 1984.

[14] E. M. Elabbasy and S. H. Saker, "Oscillation of first order neutral delay differential equations," Kyungpook Mathematical Journal, vol. 41, no. 2, pp. 311-321, 2001.

[15] K. Farrell, E. A. Grove, and G. Ladas, "Neutral delay differential equations with positive and negative coefficients," Applicable Analysis, vol. 27, no. 1-3, pp. 181-197, 1988.

[16] M. K. Grammatikopoulos, E. A. Grove, and G. Ladas, "Oscillation and asymptotic behavior of neutral differential equations with deviating arguments," Applicable Analysis, vol. 22, no. 1, pp. $1-19,1986$.

[17] J. K. Hale, Theory of Functional Differential Equations, Springer, New York, NY, USA, 1977.

[18] J. S. Yu, M.-P. Chen, and H. Zhang, "Oscillation and nonoscillation in neutral equations with integrable coefficients," Computers \& Mathematics with Applications, vol. 35, no. 6, pp. 65-71, 1998.

[19] B. G. Zhang, "Oscillation of first order neutral functionaldifferential equations," Journal of Mathematical Analysis and Applications, vol. 139, no. 2, pp. 311-318, 1989. 


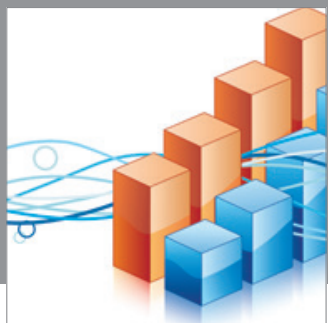

Advances in

Operations Research

mansans

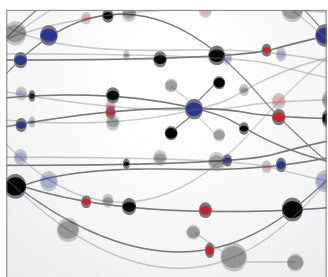

The Scientific World Journal
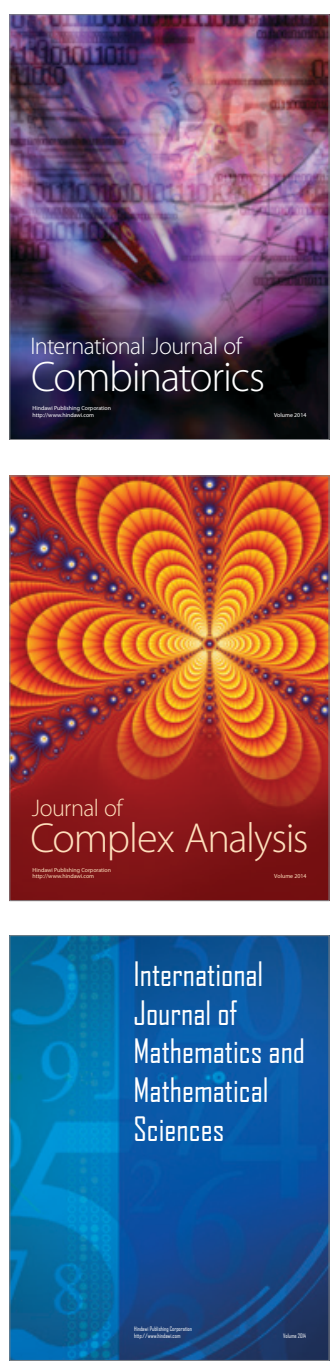
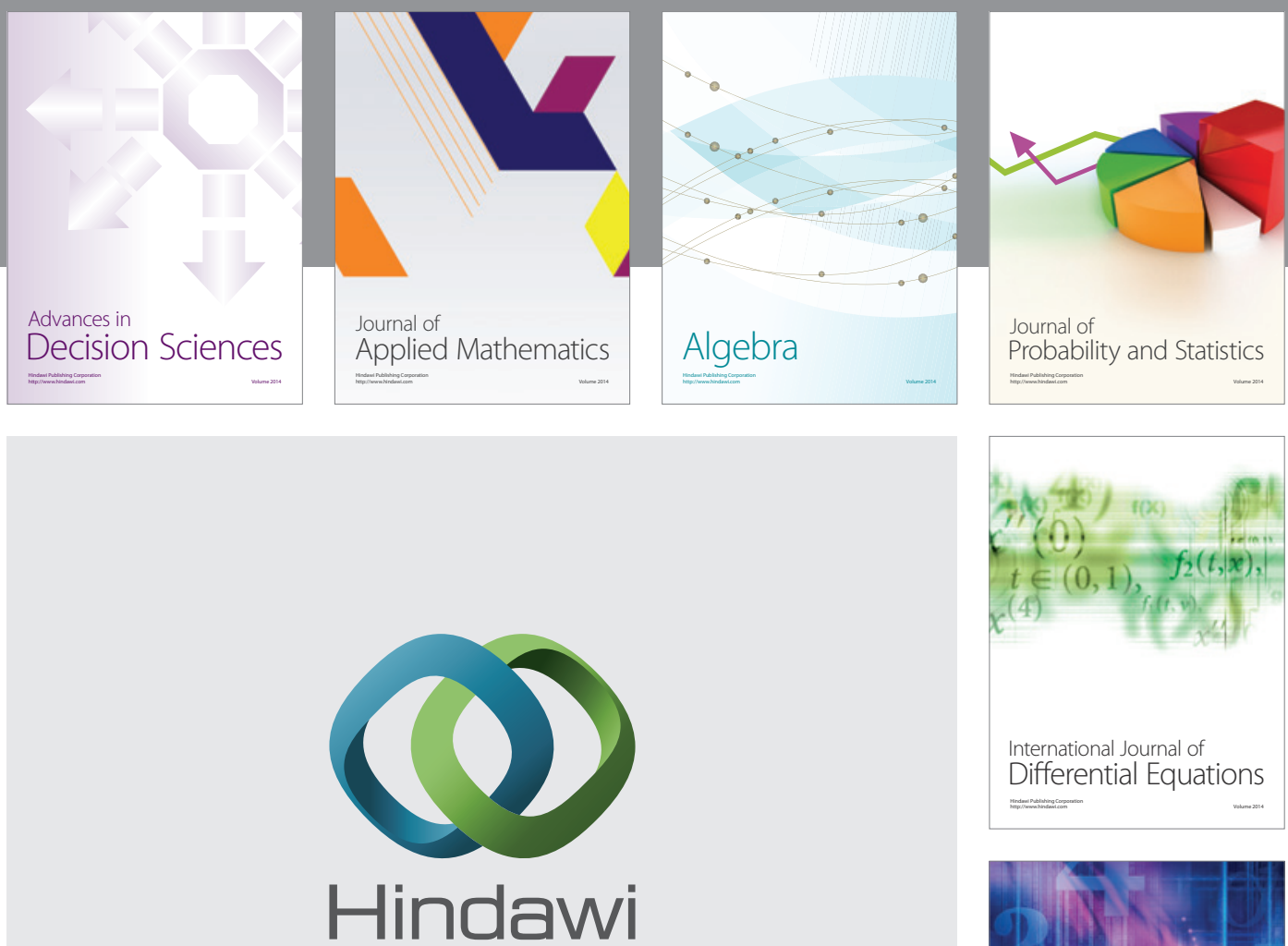

Submit your manuscripts at http://www.hindawi.com
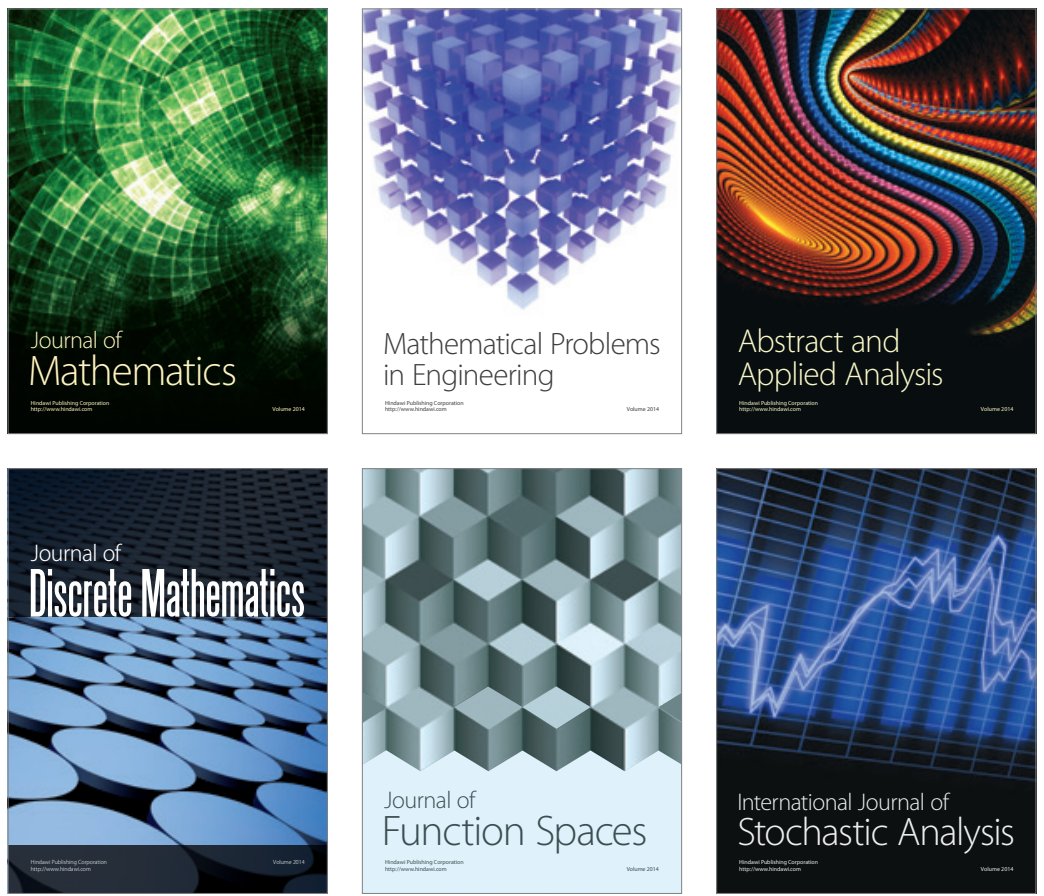

Journal of

Function Spaces

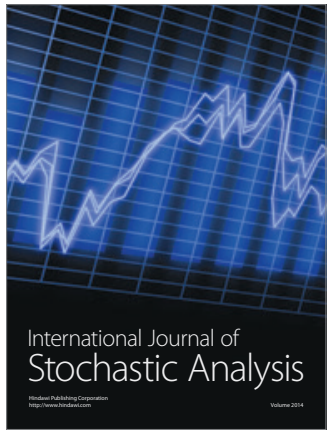

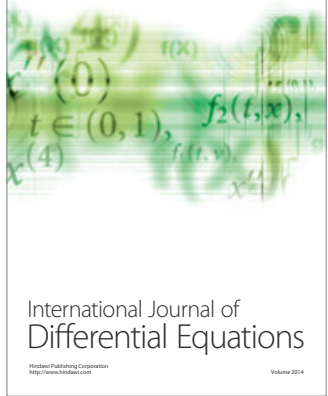
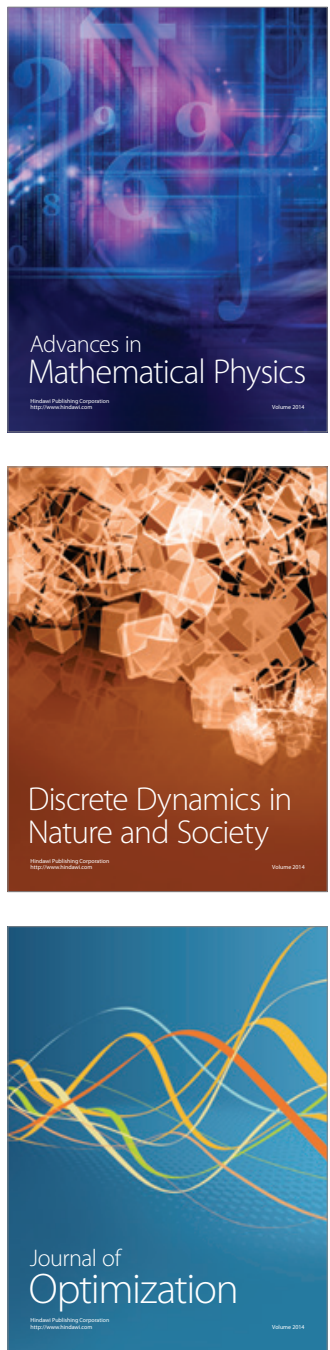